Classification

Physics Abstracts

$61.16 \mathrm{D}$

\title{
T.E.M. image of dissociated dislocation dipoles in CdTe
}

\author{
A. Orlova (*), J. P. Rivière and J. Castaing \\ Laboratoire de Physique des Matériaux, C.N.R.S., 1, place A. Briand; 92195 Meudon Cedex, France
}

(Reçu le $1^{\mathrm{er}}$ octobre 1984, révisé le 27 février 1985, accepté le $1^{\mathrm{er}}$ avril 1985)

\begin{abstract}
Résumé. - Nous avons étudié au moyen du microscope électronique en transmission, de nombreuses dislocations dissociées selon

$$
\pm \mathbf{b}=\frac{a}{2}\langle 1 \overline{1} 0\rangle \rightarrow \frac{a}{6}\langle 2 \overline{1} \overline{1}\rangle+\frac{a}{6}\langle 1 \overline{2} 1\rangle
$$

observées dans le CdTe déformé à la température ambiante [4]. Dans le présent travail nous traitons plus particulièrement le cas d'un dipôle de dislocations dissociées afin :1) de tester l'efficacité d'une méthode de dépouillement des images de dislocations dissociées en contraste cinématique [2], 2) de souligner et d'évaluer l'influence des surfaces de la lame mince sur l'équilibre de la dissociation, le CdTe étant ductile. On trouve dans cet effet l'une des causes de la dispersion des mesures de l'énergie de faute d'empilement $\gamma\{111\}, \frac{a}{6}\langle 2 \overline{1} \overline{1}\rangle$ observée dans la littérature $[4,5]$.
\end{abstract}

Abstract. - We have studied by T.E.M. several dislocations dissociated according to :

$$
\pm \mathbf{b}=\frac{a}{2}\langle 1 \overline{1} 0\rangle \rightarrow \frac{a}{6}\langle 2 \overline{1} \overline{1}\rangle+\frac{a}{6}\langle 1 \overline{2} 1\rangle
$$

which were observed in CdTe deformed at room temperature [4]. This work is dedicated to describe the case of a dipole of dissociated dislocations ; it intends to 1) check the use of a method to analyse the images of dissociated dislocations formed in kinematic contrast [2], 2) emphasize the influence of the thin foil surfaces on the dissociation equilibrium and estimate its magnitude in view of the ductility of CdTe. This seems to be at the origin of the scatter of the stacking fault energy values $\gamma\{111\}, \frac{a}{6}\langle 2 \overline{1} \overline{1}\rangle$ displayed in the literature $[4,5]$.

\section{Introduction.}

Rivière and Cadoz $[1,2]$ recently proposed a method to study the contrast of dissociated dislocations in the dark field weak beam conditions [10], defining straightforward criterions to justify the use of two beam kinematical theory to describe the contrast of dislocations [11]. Taking into account the superposition of the strain field of the partials, they established simple relations between the configuration of the dissociated dislocation and its image. This was utilized to determine the detailed geometry of the dissociated disloca-

(*) Permanent address : Institute of Physical Metallurgy, Czechosl. Acad. Science, Zizkova 22, 61662 Brno, Czechoslovakia. tion which was different from what could be deduced directly from the micrograph.

In the present paper we extend the work to the case of a dipole of edge dislocations dissociated into Shockley partials in the F.C.C. lattice,

$$
\pm \mathbf{b}=\frac{a}{2}\langle 1 \overline{1} 0\rangle \rightarrow \frac{a}{6}\langle 2 \overline{1} \overline{1}\rangle+\frac{a}{6}\langle\overline{12} 1\rangle
$$

and laying in two slip planes $\{111\}$ a distance $y$ apart (Fig. 1); the strain field of all four partials has to be combined to analyse the image contrast.

The example investigated was observed in a deformed crystal of CdTe, where dissociated dipoles are a common feature of dislocation structures [3-5]. The configuration of partials deduced from the contrast 
(a)

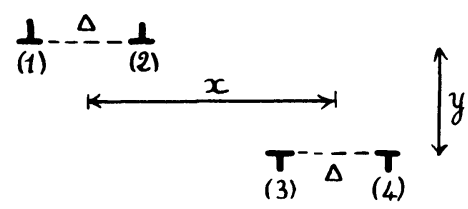

(b)
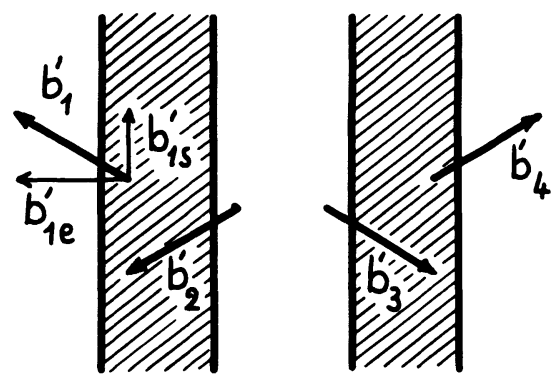

Fig. 1. - Scheme of a dissociated edge dislocation dipole : a) section by a plane perpendicular to the dislocation lines; b) projection onto the slip plane of dislocations with representation of partial Burgers vector directions $\mathbf{b}_{i}^{\prime}$ with their edge and screw component $\mathbf{b}_{i \mathrm{e}}^{\prime}$ and $\mathbf{b}_{i \mathbf{s}^{\prime}}^{\prime}$.

phenomena is discussed with respect to a change of the width of dissociation due to interaction of all the partials and the effect of the external applied stress.

\section{Contrast of dissociated dipoles observed in weak} beam T.E.M.

A summary of the method [1, 2], which will be used for the analysis of the weak beam T.E.M. contrast of a dissociated dipole, is as follows :

For simplicity, let us start from a «diffracting column " defined here as a cylinder of a crystalline matter, the diameter of which is of the order of the microscope resolution and its height is equal to the foil thickness. Its generating line is parallel to the line of intersection of the electron beam incidence plane with the diffracting planes, which are deformed in the neighbourhood of a dislocation (see the displacement formulae [9], p. 17 for instance). The crystal geometry inside the column may be related to the contrast of the dislocation within the kinematical theory [11].

In the case of $\mathbf{g} \cdot \mathbf{b} \neq 0$ the generating line possesses an inflection point at which the local distortion can be described in an isotropic elastic crystal by [1]

$$
\theta_{0}=\frac{k}{x} \cdot \frac{b}{2 \pi} \cdot \cos \beta_{1} \cdot \cos \beta_{2}
$$

where $\theta_{0}$ is the rotation of the reflecting plane, $x$ is the distance of the column from the dislocation which deforms it ; $k$ is a coefficient equal to 1 and 1.7 for the screw and edge dislocation, respectively, $\beta_{1}$ is the angle between $g$ and $b$, and $\beta_{2}$ the dislocation line inclination with respect to the plane of observation (see Fig. 2a).

Let us suppose the crystal to be deviated by $\delta \theta$ from the Bragg angle $\theta$ corresponding to the reflection $g$. Then we can always find columns which are in the

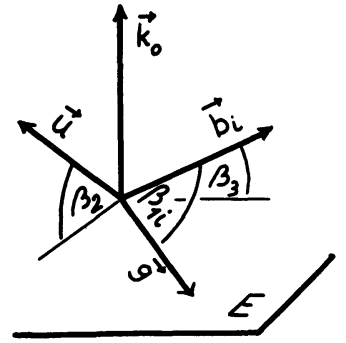

(a)

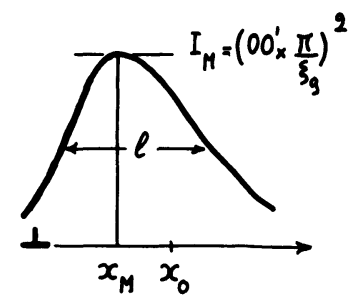

(b)
Fig. 2. - Geometrical representation of parameters used in table I [2] : a) definition of the direction of a dislocation $\mathbf{u}$, of the partial Burgers vector $\mathbf{b}_{i}$ and of the diffraction vector $\mathrm{g}$, with respect to the plane of observation $\mathrm{E} ; \mathrm{b}$ ) the intensity profile.

Bragg position at their inflection points : the distance $x_{0}$ of these columns from the dislocation line will be obtained from equation (1) by putting $\theta_{0}=\delta \theta$. If $x_{0}$ is taken as a unit, the profiles of contrast are dependent only on the dislocation character and the $(g . b)$ value [2].

Table I gives a list of parameters characterizing the profiles of contrast in units $x_{0} ; x_{M}$ is the shift of the contrast maximum with respect to the real position of the dislocation line, $l$ the width of the contrast profile and $I_{M}=\left(00^{\prime} . \pi / \xi g\right)^{2}$ the maximum intensity $\left(00^{\prime}\right.$ represents roughly the height of deformed column which is very near to the Bragg position (Fig. 2b)).

Table I. - Coefficients for the determination of contrast characteristics - the width $l$, the shift $x_{\mathrm{M}}$ of the maximum and $00^{\prime}$ a parameter proportional to the amplitude if $00^{\prime} \leqslant \xi g / 4$ [2]. For edge dislocations, the values become less accurate for $\beta_{3}>45^{\circ}$ (Fig. 2).

Screw dislocations :

\begin{tabular}{|l|l|l|l|l|}
\hline \multicolumn{1}{|c|}{$\mathbf{g} \cdot \mathbf{b}$} & 1 & 2 & 3 & 4 \\
\hline$l / x_{0}$ & 1.2 & 0.9 & 0.70 & 0.55 \\
\hline$x_{\mathrm{M}} / x_{0}$ & 0.32 & 0.50 & 0.60 & 0.66 \\
\hline$\frac{00^{\prime}}{x_{0}}$ & $\frac{2.9}{\cos \beta_{2}}$ & $\frac{2.5}{\cos \beta_{2}}$ & $\frac{2.3}{\cos \beta_{2}}$ & $\frac{2.15}{\cos \beta_{2}}$ \\
\hline
\end{tabular}

Edge dislocations :

\begin{tabular}{|l|c|c|c|}
\hline $\mathbf{g} \cdot \mathbf{b}$ & 1 & 2 & 3 \\
\hline$l / x_{0}$ & 1.3 & 0.9 & 0.7 \\
\hline$x_{\mathrm{M}} / x_{0}$ & 0.4 & 0.6 & 0.7 \\
\hline$\frac{00^{\prime}}{x_{0}}$ & $\frac{1.8}{\cos \beta_{2}}$ & $\frac{1.6}{\cos \beta_{2}}$ & $\frac{1.4}{\cos \beta_{2}}$ \\
\hline
\end{tabular}


The contrast characteristics in a particular case observed experimentally (i.e. when $\delta \theta$ is measured from the position of Kikuchi lines in the diffraction pattern) are evaluated by multiplying the appropriate coefficients from table I by $x_{0}(\delta \theta)$ given by equation (1) [2].

Let us suppose now two parallel dislocations, the dislocation $j$ situated close to the dislocation $i$ whose contrast is investigated (the dislocation may be perfect or imperfect, when $\left(\mathbf{g} \cdot \mathbf{b}_{j}\right)$ is an integer). It was shown [2] that due to the presence of dislocation $j$, all the columns contributing to the contrast of the dislocation $i$ rotate of a nearly identical angle $\theta_{i j}$ calculated from displacement formulas [9] :

$$
\theta_{i j}=\frac{k}{x_{i j}} \cdot \frac{b_{j}}{2 \pi} \cdot \cos \beta_{1, j} \cdot \cos \beta_{2} \cdot \cos ^{2} \gamma_{i, j},
$$

where $b_{j}$ is the Burgers vector of the $j$-th dislocation, $x_{i, j}$ the projected distance of dislocations $i$ and $j$ in the observation plane, $\gamma_{i j}$ the angle between the observation plane and the plane containing both dislocations $i$ and $j ; \beta_{1, j}$ and $\beta_{2}$ are defined in figure $2 \mathrm{a}$. Consequently, if there are several dislocations $j$ (perfect or imperfect) in the neighbourhood of the dislocation $i$, the total angular deviation $\delta \theta_{i}$ from the Bragg angle $\theta$, of the crystal surrounding the dislocation $i$ will be given by :

$$
\delta \theta_{i}=\delta \theta+\sum_{j} \theta_{i j}=\delta \theta+\theta_{i}
$$

By using the proportionality $I_{\mathrm{M}} \propto\left(00^{\prime}\right)^{2} \propto x_{0 i}^{2} \propto$ $\left(\delta \theta_{i}\right)^{-2}$ when $\mathbf{g} \cdot \mathbf{b}_{i}$ have the same values for all the dislocations $i$, we can determine from the contrast evolution of the successive dislocations the sense of $\mathbf{b}_{i}$ when considering the sense of $\delta \theta$ deduced from the Kikuchi line position in the diffraction pattern. This is illustrated in figure 3 for a configuration of partials forming a dissociated edge dislocation dipole, the contrast of which is analysed below. We calculate the modification of contrast for a dipole shown in figure 1 .

Let us suppose the dislocations of the dipole gliding in the planes (111) parallel to the plane of the thin foil and imaged by the diffraction vector $\overline{4} \overline{4} 0$ which is parallel to $b$. In this case all the $\frac{1}{6}\langle 2 \overline{11}\rangle$ partials give contrast $\left(\mathbf{g} \cdot \mathbf{b}^{\prime}=2\right)$ and the stacking fault is invisible. Only edge components $\mathbf{b}_{\mathbf{e}}^{\prime}$ of the partials are active in the contrast formation; they are $\mathbf{b}_{1 \mathrm{e}}^{\prime}=\mathbf{b}_{2 \mathrm{e}}^{\prime}=$ $-\mathbf{b}_{3 \mathrm{e}}^{\prime}=-\mathbf{b}_{4 \mathrm{e}}^{\prime}=\frac{1}{4}[1 \overline{1} 0]$. Equation (2) may be rewritten as

$$
\theta_{i j}=\frac{1.7 b_{j e}^{\prime}}{2 \pi x_{i j}} \cdot \cos ^{2} \gamma_{i j}
$$

We calculate now the rotation generated by dislocations $j$ at the dislocation $i$, i.e. $\theta_{i}=\sum_{j} \pm \theta_{j i}$, where the signs before $\theta_{i j}$ are related to the sense of rotations

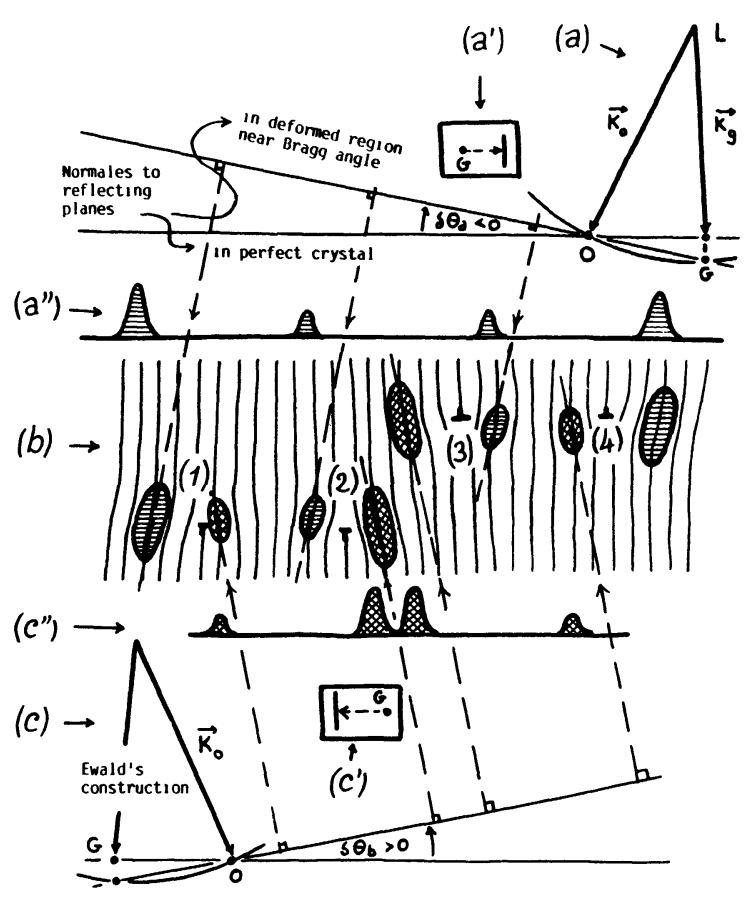

Fig. 3. - Formation of contrast of a dipole of dissociated dislocations : it is shown why in diffracting conditions described by the Ewald's constructions (a) and (c), defined by positions of the Kikuchi lines in the diffracting pattern $\left(a^{\prime}\right)$ and $\left(c^{\prime}\right)$, the profiles of contrast $\left(a^{\prime \prime}\right)$ and $\left(c^{\prime \prime}\right)$ are obtained as an image of dislocations schematized in (b), where the hatched zones correspond to the crystal volume near the Bragg position.

generated by the dislocations $j$ (see Figs. 1 and 3) :

$$
\begin{aligned}
& \theta_{1}=\theta_{21}-\theta_{31}-\theta_{41} \\
& \theta_{2}=-\theta_{12}-\theta_{32}-\theta_{42} \\
& \theta_{3}=-\theta_{13}-\theta_{23}-\theta_{43} \\
& \theta_{4}=-\theta_{14}-\theta_{24}+\theta_{34}
\end{aligned}
$$

where $\theta_{i j}=\theta_{j i}$. Using equations (4) and (5), one obtains a simple form for $\theta_{i}$ in a symmetrical case (Fig. 1) :

$$
\theta_{1}=\theta_{4}=\frac{C}{\Delta}\left[1-\frac{X}{X^{2}+Y^{2}}-\frac{(X+1)}{(X+1)^{2}+Y^{2}}\right]
$$

$$
\theta_{2}=\theta_{3}=\frac{C}{\Delta}\left[-1-\frac{X}{X^{2}+Y^{2}}-\frac{(X-1)}{(X-1)^{2}+Y^{2}}\right],
$$

where $C=1.7 b_{\mathrm{e}}^{\prime} / 2 \pi$ and $X=x / \Delta$ and $Y=y / \Delta$ are reduced coordinates with $\Delta$ the separation of partials (Fig. 1). This form is rather convenient for an estimate of the effect of the dipole configuration on the contrast observed. The terms due to interaction of partials decrease with increasing $Y$ and when $X$ is large $(X>2)$ the modification of contrast is almost independent of $Y$, i.e. of $y$. 


\section{Application to experimental observations.}

A dissociated dipole shown in figure 4 was observed in a foil of a CdTe single crystal deformed at room temperature by constant strain rate compression along the [132] direction; the final strain of $2.5 \%$ was reached at $\sigma_{\mathrm{Max}}=15 \mathrm{MPa}$. The foil was cut approximately parallel to the primary slip plane $(111) ; \overline{4} 40$ reflection type was used in the dark field weak beam conditions. For a detailed analysis, the profiles of contrast were detected by microdensitometric measurements in corresponding sections of the images. However, this detection of contrast was rather difficult because of a disturbing effect of the radiation damage produced by ion beam thinning of the foils. This effect is responsible for the intensity fluctuations and widening of the contrast seen in the micrographs. Therefore not only the maximum intensity $I_{i}^{\mathrm{E}}$ was read from the curves; also the mean intensity $\bar{I}_{i}$ within the total profile width was evaluated (Fig. 5).

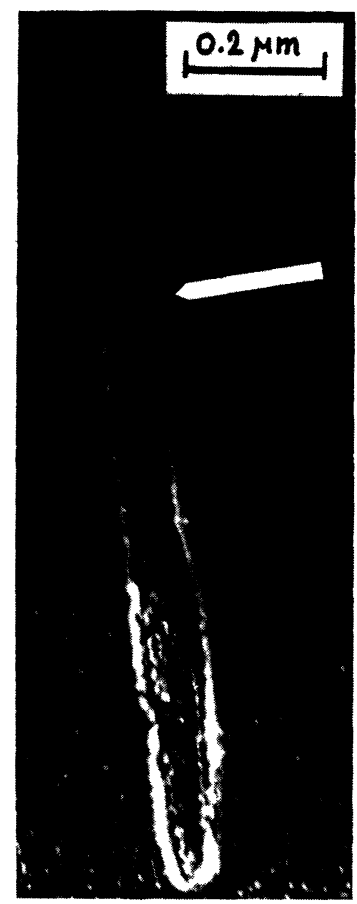

(a)
Positions of the maximum contrast and the relative intensities (Fig. 5) show a certain asymmetry of the dislocation configuration. As $x>2 \Delta$, the $\theta_{i}$ values do not vary quickly with the value of $y$ (Eq. (6)), therefore, $\delta \theta_{i}$ and the intensities are not sensitive to $y$. Moreover, the dipole should be near to equilibrium of dislocation interaction forces and thus we can suppose $x \simeq y$ for the calculation of the contrast characteristics.

The results of this calculation reported in figure 5 show a good agreement for the positions of the partials deduced from the two different diffracting conditions. They correspond to cases a) and c) illustrated in figure 3. Similarly the intensities calculated by means of $\delta \theta_{i}$ (Sect. 2) correspond rather well to the observed values. However, the calculation of $l$ gives relatively narrow lines in comparison with what is observed by microdensitometry; this is believed to be a result of the effect of radiation damage inducing fluctuations of the contrast lines as mentioned above.

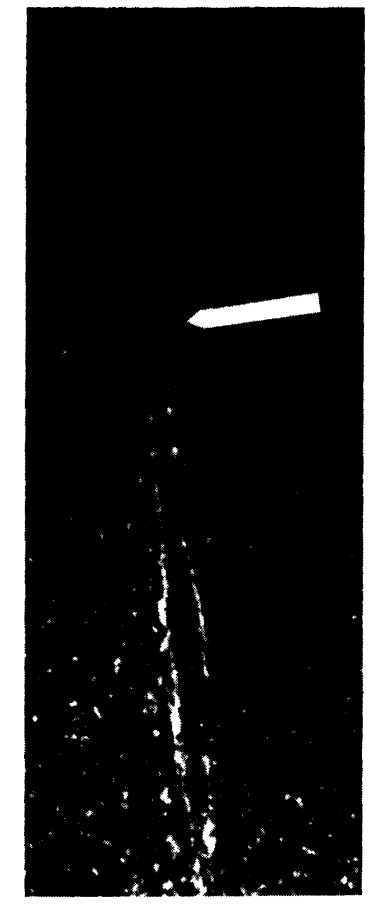

(b)
D.F. $g=4 \overline{4} 0$

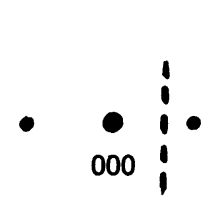

$\delta \theta_{\mathrm{a}}=-1.05 \times 10^{-2} \mathrm{rad}$.

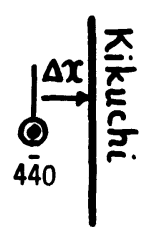

D.F. $g=\overline{4} 40$

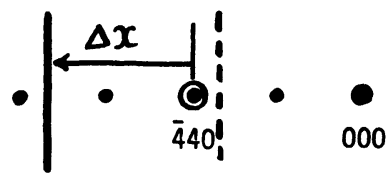

$\delta \theta_{b}=2.47 \times 10^{-2} \mathrm{rad}$.

Fig. 4. - Dissociated edge dislocation dipole in deformed CdTe. The foil plane is parallel to the (111) slip plane. Darkfield weak-beam imaging conditions corresponding to figures $3 a$ and $c$ were used in figures $4 a$ and $b$, respectively. 


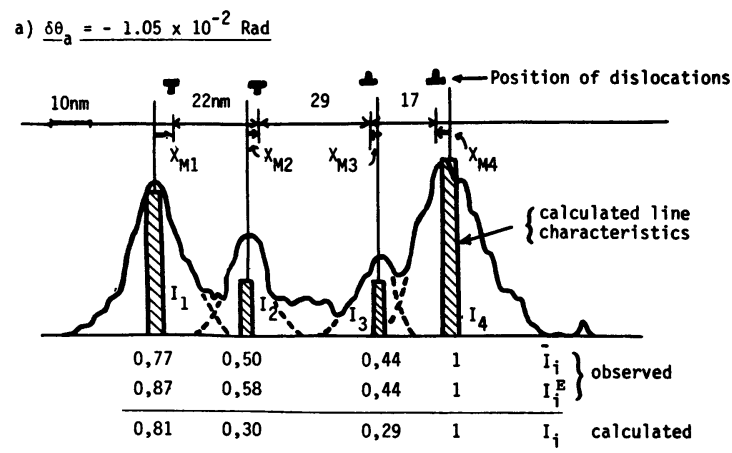

b) $\underline{\delta \theta_{b}}=2.47 \times 10^{-2} \mathrm{Rad}$

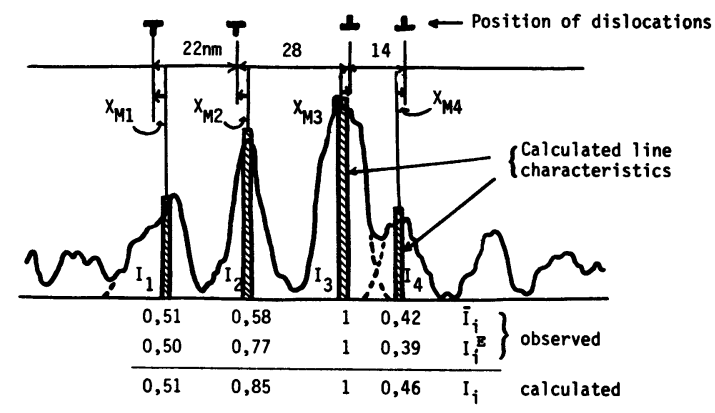

Fig. 5. - Microdensitometric curves of the profiles of contrast along the line marked in figures $4 a$ and $b$. The shifts $x_{M i}$ of real positions of dislocations with respect to the position of contrast are shown and the theoretical contrast profile (calculated by means of Table I) is schematized by rectangles of height which represents the maximum intensity $I$ and of width $l$. $I_{i}^{\mathrm{E}}$ is the experimental maximum intensity for the $i$ th partial, $\bar{I}_{i}$ the mean intensity within the total profile width (limited by dotted lines). In both cases, the greatest intensities $I_{i}, \bar{I}_{i}$ and $I_{i}^{\mathrm{E}}$ are arbitrarily taken equal to 1 . Note that the predicted widths are much smaller than the observed ones.

\section{Discussion.}

The intensities found from the microdensitometric curves $\left(I_{i}^{\mathrm{E}}\right.$ and $\left.\bar{I}_{i}\right)$ and those deduced from the diffracting conditions are both influenced by experimental errors. $I_{i}^{\mathrm{E}}, \bar{I}_{i}$ and $I_{i}$ are nearly the same (Fig. 5), so the effect of intensity fluctuations does not influence seriously the maximum intensity $I_{i}^{\mathrm{E}}$, though it increases the width of the line. Nevertheless, both $I_{i}^{\mathrm{E}}$ and $\bar{I}_{i}$ should contain an error due to the uncertainty of the background, which in the present case is increased first of all by the contrast due to the radiation damage. On the other hand, the intensities calculated from the diffraction conditions should contain the error of $\delta \theta$, which (as determined from the position of Kikuchi lines in the diffraction pattern) may reach several per cent of $\delta \theta$. However, the good correspondence of the observed and calculated intensities shows that the above errors are probably unimportant.

We did not measure accurately the separation $y$ between the slip planes. From the value of the applied stress at the end of the test $\left(\sigma_{\operatorname{Max}}=15 \mathrm{MPa}\right)$ we can deduce a maximum value for $y$, given by [6]

$$
\sigma . f=\frac{\mu b}{8 \pi(1-v) y_{\max }}
$$

$f$ is the Schmid factor, $b=0.458 \mathrm{~nm}$ and the elastic shear modulus $\mu=1.53 \times 10^{4} \mathrm{MN} / \mathrm{m}^{2}$ and Poisson's ratio $v=0.338$ [7] were used. For $y$ values lower than $y_{\max }=60 \mathrm{~nm}$ the dislocation dipoles are stable. The low sensitivity of contrast to $y$ does not cause any important error in the calculation of intensity as well as of the dislocation line positions.

Whenever the positions of the dislocation lines are known, the configuration of partials may be discussed from the point of view of the interaction forces between the partials and the stacking fault energy. The interaction forces between the partials were expressed by means of formulas of the theory of dislocations (e.g. [6], p. 83-85). The dipole equilibrium corresponds to total forces in the glide plane equal to zero for each dislocation; this can be written e.g. for dislocation (1), see figure 1:

$$
F_{12}-F_{13}-F_{14}-\gamma=0
$$

where $F_{i j}$ are interaction forces and $\gamma$ is the force resulting from the stacking fault. By varying the slip plane separation $y$ about the value of $x=47 \mathrm{~nm}$ (Fig. 1), an equilibrium dipole configuration with both dislocations dissociated was found at $y \simeq 46 \mathrm{~nm}$, which is not very different from equilibrium of perfect dislocations. The configuration of figure 4 corresponds therefore to $x \simeq y \simeq 46 \mathrm{~nm}$.

We now examine the influence of the dislocation interactions on their dissociation in order to calculate the stacking fault energy. The interaction of nonpartner partials (1), (3) or (1), (4) has a tendency to decrease the separation of partner partials (1), (2) (Fig. 1) by a force [6] $F_{13}+F_{14}=-\left(F_{23}+F_{24}\right)=$ $0.8 \times 10^{-3} \mathrm{~N} / \mathrm{m}$ and that of (3), (4) by a force $F_{31}+$ $F_{32}=-\left(F_{41}+F_{42}\right)=0.7 \times 10^{-3} \mathrm{~N} / \mathrm{m}$, these values varying very slowly with $y$ around $y=46 \mathrm{~nm}$. The interaction force between partials are $F_{12}=$ $6.9 \times 10^{-3} \mathrm{~N} / \mathrm{m}$ and $F_{34}=9.4 \times 10^{-3} \mathrm{~N} / \mathrm{m}$, respectively. The resulting interaction forces acting on dislocations (1), (2) and (3), (4) give the stacking fault energy (Fig. 1). They give values $\gamma=6.1 \pm 0.4 \mathrm{~mJ} / \mathrm{m}^{2}$ and $\gamma^{\prime}=8.7 \pm 0.9 \mathrm{~mJ} / \mathrm{m}^{2}$. Repeated measurements of the dissociation width along the dislocation line reveal fluctuations of about $1.5 \mathrm{~nm}$ which is the major cause of error.

The value of $\gamma^{\prime}$ is in good agreement with other observations on CdTe $[4,5]$, while that of $\gamma$ is lower. Depending on the existence of the elastic equilibrium, several explanations may be suggested :

(i) The large separation of dislocations (1), (2) is anomalous because the dissociated dislocation is not at equilibrium. An anomalous separation of partials may appear in their motion and has often been observed in the in situ H.V.E.M. deformation experiments [8]. 
This phenomenon in dynamical conditions is supported by the fact that under the applied stress the forces acting on the partials have slightly (about $5 \%$ ) different values due to different directions of the partial Burgers vectors. However, since our mechanical tests were performed at room temperature, it is very likely that the dipole is in equilibrium in the thin foil.

(ii) The stacking fault energy is not identical for the dissociation of two opposite dislocations in the (111) and (111) planes. However, the value of $\gamma$ is anomalously low with respect to all other observations of isolated dislocations [4], among which dissociations in both types of planes can be expected. Therefore, this explanation does not seem very probable.

(iii) Since the dipole is located in a thin foil, its equilibrium configuration may be altered by the influence of the surfaces. We have estimated a dissociation width $\Delta \sim 20 \mathrm{~nm}$ and a distance between slip planes $y \sim 46 \mathrm{~nm}$; these values are of the order of the foil thickness which is probably of about $100 \mathrm{~nm}$. If the system foil + dipole is not symmetric, there is an image force on the dislocation which is closer to the surface.

For instance, we show in the appendix that, if a dislocation is located within 12 and $25 \mathrm{~nm}$ from the surface, its dissociation width is $22 \mathrm{~nm}$ instead of $17 \mathrm{~nm}$ for a stacking fault energy of $\gamma=8.5 \mathrm{~mJ} / \mathrm{m}^{2}$. This is the easiest way to explain our observation (Figs. 4 and 5) of unequal dissociation width.

In the appendix, we also show that for such $\gamma$ values, only a few dislocations are far enough from the surface to avoid any perturbation from it. We have estimated that about $30 \%$ of the dislocations which can be observed, have their dissociation width increased by $20 \%$, thus giving too small $\gamma$ values. This image force effect contribute significantly to the scatter of experimental data for stacking fault energies $[4,5]$.

\section{Conclusion.}

The present analysis proved that the method of contrast evaluation suggested by Rivière and Cadoz [2] gives a good prediction of contrast in the case of a dissociated dipole situated in slip planes parallel to the plane of observation.

The contrast can give an information about the actual configuration of the partials, which does not correspond exactly to that observed directly in the micrographs. In the example investigated, the contrast of the partials is rather insensitive to the distance $y$ between glide planes of the dissociated dislocations.

The uneven dissociation width of the two dislocations is probably due to the image force which, in a ductile material, has to be taken into account to determine the equilibrium configuration. The effect of the surface may, indeed, increase the dissociation width; this may explain part of the scatter of stacking fault energy determinations.

\section{Acknowledgments.}

One of the authors (A. O.) worked on this problem as.a visiting scientist of the L.P.M.-C.N.R.S. in Meudon. She is greatly indebted to Professor Philibert for giving all support for her stay.

\section{Appendix.}

EQUILIBRIUM DISSOCIATION OF A DISLOCATION LOCATED CLOSE TO THE SURFACE IN A THIN FOIL. - The interaction of a dislocation and a surface can be described by using an "image dislocation" located at the mirror position outside of the solid with the opposite Burgers vector [9]. In figure 6 , we show the configuration for the dissociated dislocation (1), (2) of figure 1. Using the classical relation for the interaction forces ([6], p. 83), the component $F$ in the fault plane of the force per unit length between the partial (1) and the others (Fig. 6) is given by :

$$
\begin{array}{r}
F=\frac{\mu b_{e}^{\prime 2}}{2 \pi(1-v)}\left[\frac{1}{\Delta}+\frac{\Delta\left(h^{2}-\Delta^{2}\right)}{\left(h^{2}+\Delta^{2}\right)^{2}}\right]-\frac{\mu b_{s}^{\prime 2}}{2 \pi} \times \\
\times\left(\frac{1}{\Delta}-\frac{\Delta}{h^{2}+\Delta^{2}}\right) .
\end{array}
$$

The first term is for edge interactions and the second one for screw. Far from the surface $h \gg \Delta$, equation (7) reduces to the usual equation with only $1 / \Delta$ terms. For the edge interaction, the corrective term is always positive, since $h>\Delta$. It has the same sign for screw interaction, therefore the two terms have an additive influence giving a large change for the equilibrium dissociation of dislocations close to the surface.

At equilibrium, $\boldsymbol{F}$ is set equal to $\gamma$, the force due to stacking fault energy. Using equation (7), we have calculated the equilibrium value of $\Delta$ as a function of $h / 2$, the distance from the surface; the results are reported in figure 7, where we have used $b_{\mathrm{s}}^{\prime}=0.132 \mathrm{~nm}$ and $b_{\mathrm{e}}^{\prime}=0.229 \mathrm{~nm}$. Figure 7 shows that until fairly large depths $(\sim 30 \mathrm{~nm}) \Delta$ can be substantially larger than for a dislocation in an infinite crystal; e.g. with $\gamma=8.5 \mathrm{~mJ} / \mathrm{m}^{2}, \Delta$ is close to $22 \mathrm{~nm}$ for a dislocation between 12 and $25 \mathrm{~nm}$ from the surface, a distance

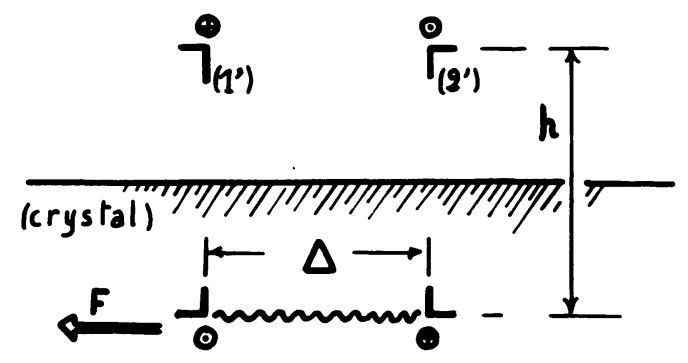

(1)

(2)

Fig. 6. - Configuration of the dissociated dislocation (1), (2) and its image $\left(1^{\prime}\right),\left(2^{\prime}\right)$. We have shown the orientation of the screw components of the Burgers vector and the positive orientation of the force $F$ applied to the partial (1). 


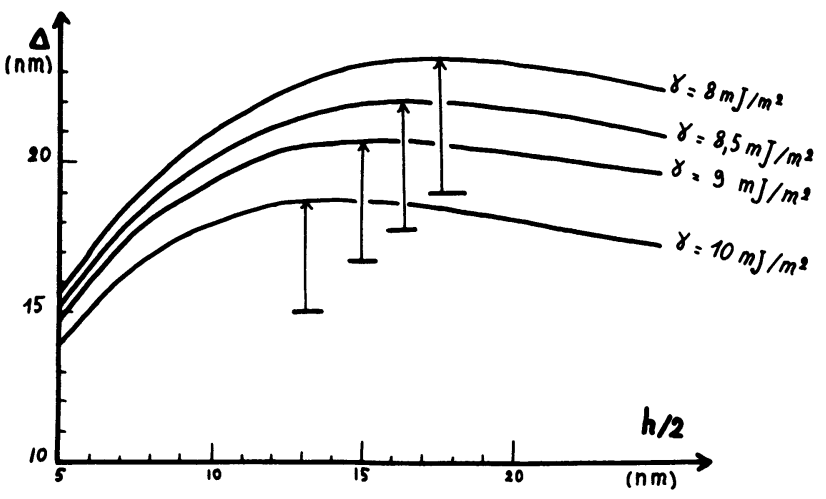

Fig. 7. - Plot of dissociation width against the distance $h / 2$ of the dissociated dislocation from the surface for various stacking fault energies $\gamma$. The horizontal bars represent the $\Delta$ values for infinite crystals ( $h$ very large) and show the importance of the modification. which covers $25 \%$ of a foil thickness of $100 \mathrm{~nm}$. This situation is likely to occur in T.E.M. studies of dissociated dislocations in ductile materials.

At his stage, we have to check that the dislocation contrasts are not modified by the surface. The analysis developed in section 2 can be applied to the dislocations and their images. The diffracting volume height which is proportional to $00^{\prime}$ has to be in the crystal. This requires :

$$
\frac{00^{\prime}}{2}<\frac{h}{2}
$$

a condition which is easily fulfilled for our observations, the distance $00^{\prime}$ being of the order of $10 \mathrm{~nm}$ with $\mathbf{g} \cdot \mathbf{b}=2$ and $\delta \theta \sim 10^{-2} \mathrm{rad}$ (Fig. 5) (Table I). The contrast of a dislocation at $5 \mathrm{~nm}$ from the surface is then heavily perturbated. At larger distances, it is less and less modified; this can be also evaluated with the process of section 2 .

\section{References}

[1] RivièRE, J. P., J. Microsc. Spectrosc. Electron 7 (1982) 121.

[2] RivièRe, J. P. and CADOZ, J., J. Microsc. Spectrosc. Electron 7 (1982) 183.

[3] Hall, E. L. and Vander Sande, J. B., J. Am. Ceram. Soc. 61 (1978) 417.

[4] Orlova, A. and Sieber, B., Acta Metall. 32 (1984) 1045.

[5] Hall, E. L. and Vander Sande, J. B., Philos. Mag. A 37 (1978) 137.

[6] HulL, D., Introduction to Dislocations (Pergamon Press) 1969.
[7] Mc Skimin, H. J. and Thomas, D. G., J. Appl. Phys. 33 (1962) 56.

[8] SuZuki, K., IChihara, M., NaKagawa, K., Maeda, K. and Takeuchi, S., Philos. Mag. A 43 (1981) 499.

[9] Friedel, J., Dislocations (Pergamon Press) 1964.

[10] Cockayne, D. J. H., RaY, I. L. F., Whelan, M. J., Philos. Mag. 20 (1969) 1265.

[11] Hirsch, P. B., Howie, A., Whelan, M. J., Philos. Trans. R. Soc. A 252 (1960) 499. 